\title{
ROTIV: RFID Ownership Transfer with Issuer Verification
}

\author{
Kaoutar Elkhiyaoui, Erik-Oliver Blass, and Refik Molva \\ Eurecom, Sophia-Antipolis, France \\ \{kaoutar.elkhiyaoui, erik-oliver.blass,refik.molva\}@eurecom.fr
}

\begin{abstract}
RFID tags travel between partner sites in a supply chain. For privacy reasons, each partner owns the tags present at his site, i.e., the owner is the only entity able to authenticate his tags. When passing tags on to the next partner in the supply chain, ownership of the old partner is transferred to the new partner. In this paper, we propose ROTIV, a protocol that allows secure ownership transfer against malicious owners. ROTIV offers as well issuer verification to prevent malicious partners from injecting fake tags not originally issued by some trusted party. As part of ownership transfer, ROTIV provides a constant-time, privacypreserving authentication. ROTIV's main idea is to combine an HMACbased authentication with public key encryption to achieve constant time authentication and issuer verification. To assure privacy, ROTIV implements key update techniques and tag state re-encryption techniques, performed on the reader. ROTIV is especially designed for lightweight tags which are only required to evaluate a hash function.
\end{abstract}

\section{Introduction}

Supply chain management is one of the main applications of RFID tags today. Each RFID tag is physically attached to a product to allow product tracking and inventorying. As products travel in a supply chain, their ownership is transferred from one supply chain partner to another, and so is the ownership of their corresponding RFID tags. Tag ownership in this setting is the capability that allows an owner of tag $T$ to authenticate, access, and transfer the ownership of $T$. Generally, the supply chain partners are reluctant into sharing their private information. Therefore, each partner requires to be the only authorized entity that can interact with tags in his site. To that effect, tags and partners in the supply chain must implement a secure ownership transfer protocol.

A secure ownership transfer protocol should fulfill two main security requirements: 1) mutual authentication between the owner of a $\operatorname{tag} T$ (partner in the supply chain) and tag $T$ to tell apart legitimate tags from counterfeits. 2) exclusive ownership: non-authorized parties must not be able to transfer the ownership of $\operatorname{tag} T$ without the consent of $T$ 's owner. Furthermore, ownership transfer must be privacy preserving. It must ensure 1) tag backward unlinkability: ownership transfer has to prevent the previous owner of a tag from tracing a tag once he releases its ownership, see Lim and Kwon [13]. 2) tag forward unlinkability: 
ownership transfer must prevent the new owner of a tag from tracing the tag's past interactions.

In addition to the basic features of tag ownership transfer as previously addressed in [14, 13, 7, 18], this paper proposes an efficient ownership transfer protocol that also allows a party possessing the right references to verify the issuer of a tag. A possible scenario for issuer verification is a supply chain where partners want to check that a product originates from a trusted partner.

An efficient ownership transfer protocol calls for an efficient authentication protocol. Current RFID authentication schemes based on symmetric cryptographic primitives require at least a logarithmic cost in the number of tags, see Burmester et al. 4]. Previously proposed tag/reader authentication protocols that achieve constant time authentication rely on public key cryptography performed on the tag as in [12]. However, RFID tags are constrained devices that cannot implement asymmetric cryptography.

The above schemes are designed to be privacy preserving against a strong adversary as defined by Juels and Weis [9], who can continuously eavesdrop on tags' communications. We claim that such an adversary is unrealistic in distributed supply chains which is the targeted setting by ROTIV. In ROTIV, we relax some privacy requirements to achieve mutual authentication in constant time while the tag performs only symmetric cryptographic operations (hash functions).

In ROTIV, a tag $T$ stores in addition to its symmetric key, a public key encryption of its identification information computed by $T$ 's owner. The public key encryption helps the owner to identify the $\operatorname{tag} T$ first, then the symmetric key is used to authenticate both $T$ and its current owner. In order to ensure tag privacy, we update $T$ 's state after each successful authentication. Moreover, each tag $T$ in ROTIV is associated with a set of ownership references. $T$ 's ownership references allow $T$ 's owner to authenticate $T$ and to transfer $T$ 's ownership. Finally, to allow tag issuer verification by third parties, a tag $T$ stores an encryption of the issuer's signature. Provided with some trapdoor information from $T$ 's owner( the randomness used to encrypt $\mathcal{I}$ 's signature), a third party verifier can verify whether the signature stored on $T$ corresponds to a legitimate issuer or not.

In summary, ROTIV's contributions are:

- ownership transfer that ensures both tag forward unlinkability against the tag's new owner and tag backward unlinkability against the tag's previous owner.

- a privacy-preserving, and constant time authentication while tags are only required to compute a hash function.

- contrary to related work [17, 14, 7, 11], ROTIV does not require a trusted third party to perform tag ownership transfer.

- issuer verification protocol that allows prospective owners of a $\operatorname{tag} T$ to check the identity of the party issuing $T$.

- formal definitions of privacy and security requirements of tag ownership transfer.

- formal proofs of ROTIV security and privacy. 


\section{RFID Ownership Transfer with Issuer Verification}

An ownership transfer protocol with issuer verification involves the following entities.

\section{$2.1 \quad$ Entities}

- Tags $T_{i}$ : Each tag is attached to a single item. A tag $T_{i}$ has a re-writable memory representing $T_{i}$ 's current state $s_{(i, j)}$ at time $j$. Tags can compute hash function $G$. $\mathcal{T}$ denotes the set of legitimate tags $T_{i}$.

- Issuer $\mathcal{I}$ : The issuer $\mathcal{I}$ initializes tags and attaches each tag $T_{i}$ to a product. For each tag $T_{i}, \mathcal{I}$ creates a set ownership references $\operatorname{ref}_{T_{i}}^{O}$ that he gives to $T_{i}$ 's owner. $\mathcal{I}$ writes an initial state $s_{(i, 0)}$ into $T_{i}$.

- Owner $O_{\left(T_{i}, k\right)}$ : Is the owner of a tag $T_{i}$ at time $k . O_{\left(T_{i}, k\right)}$ stores a set of ownership references $\operatorname{ref}_{T_{i}}^{O}$ that allows him to authenticate tags $T_{i}$ and to transfer $T_{i}$ 's ownership to a new owner. (O) denotes the set of all owners $O_{\left(T_{i}, k\right)}$. An owner $O_{\left(T_{i}, k\right)}$ comprises a database $\mathcal{D}_{k}$ and an RFID reader $R_{k}$.

- Verifier $\mathcal{V}$ : Before accepting the ownership of some tag $T_{i}$, any prospective owner $O_{\left(T_{i}, k+1\right)}$ wants to verify the identity of tag $T_{i}$ 's issuer, therewith becoming a verifier $\mathcal{V}$. Owner $O_{\left(T_{i}, k\right)}$ of $T_{i}$ provides $\mathcal{V}$ with verification references $\operatorname{ref}_{T_{i}}^{V}$ allowing $\mathcal{V}$ to verify the identity of the issuer of $T_{i}$.

\subsection{RFID Ownership Transfer with Issuer Verification}

Secure ownership transfer raises four major requirements as follows:

1.) During daily operations, current owner $O_{\left(T_{i}, k\right)}$ of $\operatorname{tag} T_{i}$ in the supply chain has to be able to perform a number of mutual authentications with $T_{i}$.

2.) Eventually, $O_{\left(T_{i}, k\right)}$ has to pass $T_{i}$ to the next owner $O_{\left(T_{i}, k+1\right)}$ in the supply chain. Therefore, $O_{\left(T_{i}, k\right)}$ and $O_{\left(T_{i}, k+1\right)}$ must exchange the ownership references.

3.) Once previous owner $O_{\left(T_{i}, k\right)}$ releases ownership of a tag $T_{i}$, new owner $O_{\left(T_{i}, k+1\right)}$ must securely update any secrets stored on $T_{i}$, such that only $O_{\left(T_{i}, k+1\right)}$ is able to authenticate $T_{i}$ and eventually pass $T_{i}$ to the next owner $O_{\left(T_{i}, k+2\right)}$.

4.) Before accepting tag ownership, a prospective owner $O_{\left(T_{i}, k+1\right)}$, has to perform issuer verification. That is, upon receipt of $T_{i}$ verification references $\operatorname{ref}_{T_{i}}^{V}$ from $T_{i}$ 's current owner, $O_{\left(T_{i}, k+1\right)}$ is able to verify whether $T_{i}$ has been originally issued by $\mathcal{I}$.

\section{Problem Statement}

Recently proposed protocols on RFID tag ownership transfer [13, 7, 18] rely on symmetric primitives to perform privacy preserving mutual authentication and secure ownership transfer. As depicted in Figure 1, a tag $T_{i}$ in these protocols

- stores a state $s_{(i, j)}=k_{(i, j)}$. This state corresponds to a secret key which is shared between $T_{i}$ and $T_{i}$ 's owner $O_{\left(T_{i}, k\right)}$. 


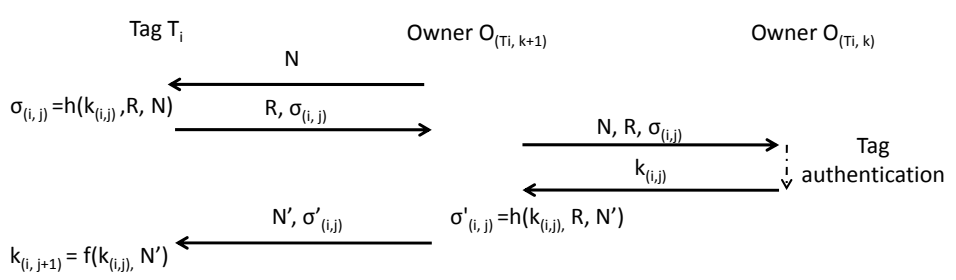

Fig. 1. Ownership transfer protocol

- computes a secure symmetric primitive $h$ that is used to authenticate mutually $T_{i}$ and $O_{\left(T_{i}, k\right)}$ using the secret key $k_{(i, j)}$.

- computes a function $f$ that is used to update the secret key of $T_{i}$ after a successful mutual authentication.

However, such protocols suffer from inherent limitations:

1) Linear complexity: As previously proposed protocols in [13, 18, 11] use symmetric primitives to authenticate a tag $T_{i}$, an owner has to try all the tags' keys in his database to authenticate $T_{i}$. Thus, in these schemes the authentication takes a linear time in the number of tags.

2) Denial of service: To ensure forward unlinkability, tag $T_{i}$ updates its key $k_{(i, j)}$ using a secure hash function $g$ even if the authentication with its owner $O_{\left(T_{i}, k\right)}$ is not successful as shown by Ohkubo et al. [15]. Also, $O_{\left(T_{i}, k\right)}$ keeps a limited set of $\eta$ keys $\left(k_{(i, j+1)}, k_{(i, j+2)}, \ldots, k_{(i, j+\eta)}\right)=\left(g\left(k_{(i, j)}\right), g^{2}\left(k_{(i, j)}\right), \ldots, g^{\eta}\left(k_{(i, j)}\right)\right)$ in his database $\mathcal{D}_{k}$ after each successful authentication with $T_{i}$. Thus, $O_{\left(T_{i}, k\right)}$ will still be able to authenticate $T_{i}$ even if the authentication fails up to $\eta-1$ times. However, an adversary can query $T_{i}$ up to $p>\eta$ times, and therefore desynchronize $T_{i}$ and $O_{\left(T_{i}, k\right)}$.

3) No tag issuer verification: Without tag issuer verification, owners and therewith partners in the supply chain will be able to inject tags that were not issued by trusted parties. We claim that in the real world, the prospective owner of tag $T_{i}$ will require verifying the origin of $T_{i}$ before accepting it.

To cope with these limitations we propose ROTIV. To achieve constant time authentication, a tag $T_{i}$ in ROTIV stores in addition to its symmetric key $k_{(i, j)}$, an Elgamal ciphertext $c_{(i, j)}$ of $T_{i}$ 's identification information. When $T_{i}$ is queried, it replies with $c_{(i, j)}$ and an HMAC computed using $k_{(i, j)}$. The owner decrypts $c_{(i, j)}$ and identifies $T_{i}$. Once $T_{i}$ is identified, the owner authenticates $T_{i}$ through HMAC. Furthermore, to prevent denial of service, a tag in ROTIV does not update its symmetric key unless the authentication is successful. Finally, to provide tag issuer verification, the ciphertext $c_{(i, j)}$ encrypts the signature of $T_{i}$ 's identifier by the issuer.

Note that protocols presented above [13, 7, 18] are designed to be forward privacy preserving against a strong adversary that continuously monitors tags [9, 19, 16]. However, in order to achieve both constant time authentication and denial of service resistance while the tag only computes hash functions, ROTIV must consider a more realistic adversary model. The adversary cannot continuously monitor a tag, i.e., there is at least one communication between the tag and its owner that is unobserved by the adversary. 
Hence, ROTIV defines new privacy and security requirements that will be further discussed in Section 5. These requirements are along the same lines as recent research on RFID security such as [9, 19, 16].

Now, we present ROTIV in $\$ 4$ followed by our privacy and security models in 95 .

\section{ROTIV}

ROTIV takes place in subgroups of elliptic curves that support bilinear pairings.

\subsection{Preliminaries}

Bilinear pairing. Let $\mathbb{G}_{1}, \mathbb{G}_{2}$ and $\mathbb{G}_{T}$ be groups, such that $\mathbb{G}_{1}$ and $\mathbb{G}_{T}$ have the same prime order $q$. Pairing $e: \mathbb{G}_{1} \times \mathbb{G}_{2} \rightarrow \mathbb{G}_{T}$ is a bilinear pairing if:

1. $e$ is bilinear $: \forall x, y \in \mathbb{Z}_{q}, g_{1} \in \mathbb{G}_{1}$ and $g_{2} \in \mathbb{G}_{2}, e\left(g_{1}^{x}, g_{2}^{y}\right)=e\left(g_{1}, g_{2}\right)^{x y}$;

2. $e$ is computable: there is an efficient algorithm to compute $e\left(g_{1}, g_{2}\right)$ for any $\left(g_{1}, g_{2}\right) \in \mathbb{G}_{1} \times \mathbb{G}_{2} ;$

3. $e$ is non-degenerate: if $g_{1}$ is a generator of $\mathbb{G}_{1}$ and $g_{2}$ is a generator of $\mathbb{G}_{2}$, then $e\left(g_{1}, g_{2}\right)$ is a generator of $\mathbb{G}_{T}$.

ROTIV's security and privacy rely on two assumptions.

Definition 1 (BCDH Assumption). Let $g_{1}$ be a generator of $\mathbb{G}_{1}$ and $g_{2}$ be a generator of $\mathbb{G}_{2}$. We say that the BCDH assumption holds if, given $g_{1}, g_{1}^{x}, g_{1}^{y}, g_{1}^{z} \in$ $\mathbb{G}_{1}$ and $g_{2}, g_{2}^{x}, g_{2}^{y} \in \mathbb{G}_{2}$ for random $x, y, z \in \mathbb{F}_{q}$, the probability to compute $e\left(g_{1}, g_{2}\right)^{x y z}$ is negligible.

Definition 2 (SXDH Assumption). The $S X D H$ assumption holds if $\mathbb{G}_{1}$ and $\mathbb{G}_{2}$ are two groups with the following properties:

1. There exists a bilinear pairing $e: \mathbb{G}_{1} \times \mathbb{G}_{2} \rightarrow \mathbb{G}_{T}$.

2. The decisional Diffie-Hellman problem $(D D H)$ is hard in both $\mathbb{G}_{1}$ and $\mathbb{G}_{2}$.

Thus, ROTIV uses bilinear groups where DDH is hard, see Ballard et al. [3], Ateniese et al. [1, 2]. Such groups can be chosen as specific subgroups of MNT curves. Also, results by Galbraith et al. [8] indicate the high efficiency of such pairings.

\subsection{ROTIV Description}

1. Overview In ROTIV, a tag $T_{i}$ stores a state $s_{(i, j)}=\left(k_{(i, j)}, c_{(i, j)}\right)$, where $k_{(i, j)}$ is a key shared with the owner of $T_{i}$, and $c_{(i, j)}$ is an Elgamal encryption of $T_{i}$ 's identification information.

When an owner $O_{\left(T_{i}, k\right)}$ starts a mutual authentication with $T_{i}, T_{i}$ replies with $c_{(i, j)}$ along with an HMAC computed using $T_{i}$ 's secret key $k_{(i, j)}$. Upon receipt of $c_{(i, j)}, O_{\left(T_{i}, k\right)}$ uses his Elgamal secret key to decrypt $c_{(i, j)}$. After decryption, $O_{\left(T_{i}, k\right)}$ checks if the resulting plaintext is in his database $\mathcal{D}_{k}$. If so, $O_{\left(T_{i}, k\right)}$ looks 
up the symmetric key $k_{(i, j)}$ of tag $T_{i}$ in his database and verifies the HMAC sent by $T_{i}$. Therefore, ROTIV allows for mutual authentication with tag $T_{i}$ in constant time, while the tag is only required to compute a symmetric primitive, i.e., HMAC.

To perform ownership transfer of tag $T_{i}$, the current owner $O_{\left(T_{i}, k\right)}$ of $T_{i}$ gives $O_{\left(T_{i}, k+1\right)} T_{i}$ 's ownership references $\operatorname{ref}_{T_{i}}^{O}$ that will be used by $O_{\left(T_{i}, k+1\right)}$ to authenticate himself to $T_{i}$ and to update $T_{i}$ 's state.

In order to ensure $T_{i}$ 's forward and backward privacy, the owner $O_{\left(T_{i}, k\right)}$ of $T_{i}$ updates the ciphertext stored on $T_{i}$ in every authentication he runs with $T_{i}$, using Elgamal re-encryption mechanisms. Moreoever, $T_{i}$ updates its key $k_{(i, j)}$ after each successful authentication.

Finally, to achieve tag issuer verification, the ciphertext $c_{(i, j)}$ stored on $T_{i}$ encrypts a signature of $\mathcal{I}$ on $T_{i}$ 's identifier. To perform issuer verification for tag $T_{i}$, a verifier $\mathcal{V}$ is provided with the ciphertext $c_{(i, j)}$ stored in $T_{i}$ along with some trapdoor information called verification references $\operatorname{ref}_{T_{i}}^{V}$. Then, given $c_{(i, j)}$ and $\operatorname{ref}_{T_{i}}^{V}, \mathcal{V}$ is able to verify if $c_{(i, j)}$ is an encrypted signature by $\mathcal{I}$ of $T_{i}$ 's identifier.

2. Description A ROTIV system comprises $l$ owners $O_{\left(T_{i}, k\right)}$ and $n \operatorname{tags} T_{i}$. Each $\operatorname{tag} T_{i}$ can evaluate a cryptographic hash function $G$ to compute an HMAC. The HMAC is used to authenticate $T_{i}$ and $T_{i}$ 's owner, and to update the symmetric key after each successful authentication.

In the rest of this section we use the notation $\operatorname{HMAC}_{k}\left(m, m^{\prime}\right)=$ $\mathrm{HMAC}_{\mathrm{k}}\left(\mathrm{m} \| \mathrm{m}^{\prime}\right)$, where $\|$ denotes concatenation.

Setup. The issuer $\mathcal{I}$ outputs $\left(q, \mathbb{G}_{1}, \mathbb{G}_{2}, \mathbb{G}_{T}, g_{1}, g_{2}, e\right)$, where $\mathbb{G}_{1}, \mathbb{G}_{T}$ are subgroups of prime order $q, g_{1}$ and $g_{2}$ are random generators of $\mathbb{G}_{1}$ and $\mathbb{G}_{2}$ respectively, and $e: \mathbb{G}_{1} \times \mathbb{G}_{2} \rightarrow \mathbb{G}_{T}$ is a bilinear pairing. The issuer chooses $x \in \mathbb{Z}_{q}^{*}$ and computes $g_{2}^{x}$. $\mathcal{I}$ 's secret key is $s k=x$ and his public key is $p k=g_{2}^{x}$.

For each owner $O_{\left(T_{i}, k\right)} \mathcal{I}$ randomly selects $\alpha_{k} \in \mathbb{Z}_{q}^{*}$ and computes the pair $\left(g_{1}^{\alpha_{k}^{2}}, g_{2}^{\alpha_{k}}\right)$. The system supplies each owner $O_{\left(T_{i}, k\right)}$ with his secret key $s k=\alpha_{k}$ and his public key $p k=\left(g_{1}^{\alpha_{k}^{2}}, g_{2}^{\alpha_{k}}\right)$. All owners know each other's public key.

Tag Initialization. The issuer $\mathcal{I}$ initializes a tag $T_{i}$ owned by $O_{\left(T_{i}, k\right)} . \mathcal{I}$ picks a random number $t_{i} \in \mathbb{F}_{q}$. Using a cryptographic hash function $H: \mathbb{F}_{q} \rightarrow \mathbb{G}_{1}, \mathcal{I}$ computes $h_{i}=H\left(t_{i}\right) \in \mathbb{G}_{1}$. Then, $\mathcal{I}$ computes $u_{(i, 0)}=1$ and $v_{(i, 0)}=h_{i}^{x}$. Finally, $\mathcal{I}$ chooses randomly a key $k_{(i, 0)} \in \mathbb{F}_{q}$. Tag $T_{i}$ stores: $s_{(i, 0)}=\left(k_{(i, 0)}, c_{(i, 0)}\right)$, where $c_{(i, 0)}=\left(u_{(i, 0)}, v_{(i, 0)}\right) . \mathcal{I}$ gives $O_{\left(T_{i}, k\right)}$ the tag $T_{i}$ and the corresponding ownership references $\operatorname{ref}_{T_{i}}^{O}=\left(k_{i}^{\text {old }}, k_{i}^{\text {new }}, x_{i}, y_{i}\right)=\left(k_{(i, 0)}, k_{(i, 0)}, t_{i}, h_{i}^{x}\right)$.

Before accepting the tag, $O_{\left(T_{i}, k\right)}$ reads $T_{i}$ and checks if the ownership references verify the equation: $e\left(H\left(x_{i}\right), g_{2}^{x}\right)=e\left(y_{i}, g_{2}\right)$. If so, this implies that $T_{i}$ is actually issued by $\mathcal{I}$, that is $y_{i}=H\left(x_{i}\right)^{x}$.

The owner $O_{\left(T_{i}, k\right)}$ adds an entry $E_{T_{i}}$ for tag $T_{i}$ in his database $\mathcal{D}_{k}: E_{T_{i}}=$ $\left(y_{i}, \operatorname{ref}_{T_{i}}^{O}\right) . y_{i}$ acts as the index of $T_{i}$ in $O_{\left(T_{i}, k\right)}$ 's database $\mathcal{D}_{k}$. Once the owner 
$\operatorname{Tag} \mathrm{T}_{\mathrm{i}}$

\begin{tabular}{|c|}
\hline 1. $\mathrm{N}$ \\
\hline 2. $\sigma_{(i, j)}, c_{(i, j)}, R_{(i, j)}$ \\
\hline 3. $\sigma_{(i, j)}^{\prime}, c_{(i, j+1)}$ \\
\hline
\end{tabular}

Owner $\mathrm{O}_{(\mathrm{Ti}, \mathrm{k})}$

Fig. 2. Authentication in ROTIV

$O_{\left(T_{i}, k\right)}$ accepts the tag, he overwrites its content. He chooses randomly $r_{(i, 1)} \in \mathbb{F}_{q}$ and computes an Elgamal encryption of $y_{i}$ using his public key $g_{1}^{\alpha_{k}^{2}}: c_{(i, 1)}=$ $\left(u_{(i, 1)}, v_{(i, 1)}\right)=\left(g_{1}^{r_{(i, 1)}}, y_{i} g_{1}^{\alpha_{k}^{2} r_{(i, 1)}}\right)$. Therefore, $s_{(i, 1)}=\left(k_{(i, 1)}=k_{(i, 0)}, c_{(i, 1)}\right)$.

Authentication protocol. To authenticate a tag $T_{i}$, the owner $O_{\left(T_{i}, k\right)}$ decrypts the ciphertext $c_{(i, j)}=\left(u_{(i, j)}, v_{(i, j)}\right)$ sent by $T_{i}$ and gets $y_{i}$. Using $y_{i}$, $O_{\left(T_{i}, k\right)}$ identifies $T_{i}$ and starts a hash-based mutual authentication. If the mutual authentication succeeds, both the owner $O_{\left(T_{i}, k\right)}$ and the $\operatorname{tag} T_{i}$ update their keys.

1. To start an authentication with tag $T_{i}$, the owner $O_{\left(T_{i}, k\right)}$ sends a random nonce $N$ to $T_{i}$ as depicted in Figure 2 .

Once $T_{i}$ receives $N$, it generates a random number $R_{(i, j)} \in \mathbb{F}_{q}$. Using its secret key $k_{(i, j)}, T_{i}$ computes: $\sigma_{(i, j)}=\operatorname{HMAC}_{k_{(i, j)}}\left(N, R_{(i, j)}, c_{(i, j)}\right)$. This HMAC serves two purposes, it authenticates $T_{i}$ and ensures the integrity of the message sent by $T_{i}$.

2. $T_{i}$ replies with $\left(R_{(i, j)}, c_{(i, j)}=\left(u_{(i, j)}, v_{(i, j)}\right), \sigma_{(i, j)}\right)$.

Upon receiving $T_{i}$ 's reply, the owner $O_{\left(T_{i}, k\right)}$ decrypts $c_{(i, j)}$ using his secret key $\alpha_{k}$ and gets $y_{i}=\frac{v_{(i, j)}}{\left(u_{(i, j)}\right)^{\alpha_{k}^{2}}} . O_{\left(T_{i}, k\right)}$ checks if $y_{i} \in \mathcal{D}_{k}$. If not, $O_{\left(T_{i}, k\right)}$ aborts authentication. Otherwise, $O_{\left(T_{i}, k\right)}$ looks up $T_{i}$ 's ownership references $\operatorname{ref}_{T_{i}}^{O}=$ $\left(k_{i}^{\text {old }}, k_{i}^{\text {new }}, t_{i}, h_{i}^{x}\right)$ in $\mathcal{D}_{k}$ and checks if: $\sigma_{(i, j)}=\operatorname{HMAC}_{k_{i}^{\text {new }}}\left(N, R_{(i, j)}, c_{(i, j)}\right)$ or $\sigma_{(i, j)}=\operatorname{HMAC}_{k_{i}^{\text {old }}}\left(N, R_{(i, j)}, c_{(i, j)}\right)$. If not, $O_{\left(T_{i}, k\right)}$ aborts authentication. If $\operatorname{HMAC}_{k_{i}^{\text {old }}}\left(N, R_{(i, j)}, c_{(i, j)}\right)=\sigma_{(i, j)}$ then $k_{(i, j)}=k_{i}^{\text {old }}$, otherwise $k_{(i, j)}=k_{i}^{\text {new }}$. $O_{\left(T_{i}, k\right)}$ chooses a new random number $r_{(i, j+1)} \in \mathbb{F}_{q}^{*}$ and computes:

$$
\begin{aligned}
c_{(i, j+1)} & =\left(u_{(i, j+1)}, v_{(i, j+1)}\right)=\left(g_{1}^{r_{(i, j+1)}}, y_{i} g_{1}^{\alpha_{k}^{2} r_{(i, j+1)}}\right) \\
\sigma_{(i, j)}^{\prime} & =\operatorname{HMAC}_{k_{(i, j)}}\left(R_{(i, j)}, c_{(i, j+1)}\right)
\end{aligned}
$$

Finally, $O_{\left(T_{i}, k\right)}$ updates the symmetric keys $k_{i}^{\text {old }}$ and $k_{i}^{\text {new }}$ in his database $\mathcal{D}_{k}$ : $\left(k_{i}^{\text {old }}, k_{i}^{\text {new }}\right)=\left(k_{(i, j)}, G\left(k_{(i, j)}, N\right)\right)$.

3. $O_{\left(T_{i}, k\right)}$ sends $c_{(i, j+1)}$ and $\sigma_{(i, j)}^{\prime}$ to $T_{i}$.

Once $T_{i}$ receives $\sigma_{(i, j)}^{\prime}$ and $c_{(i, j+1)}$, it checks if $\sigma_{(i, j)}^{\prime}=$ $\mathrm{HMAC}_{k_{(i, j)}}\left(R_{(i, j)}, c_{(i, j+1)}\right)$. If not $T_{i}$ aborts authentication. Otherwise, $T_{i}$ updates its key such that $k_{(i, j+1)}=G\left(k_{(i, j)}, N\right)$ and rewrites its state $s_{(i, j+1)}=\left(k_{(i, j+1)}, c_{(i, j+1)}\right)$. 


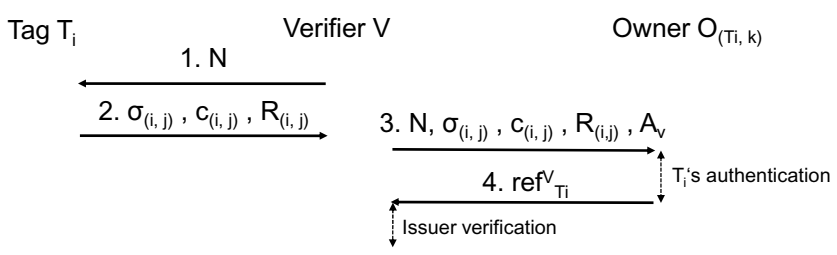

Fig. 3. Issuer verification in ROTIV

Desynchronization. If the last message of the authentication protocol is lost, tag $T_{i}$ will not update its state and therewith, $T_{i}$ will not update its symmetric key $k_{(i, j)}$. However, as the owner $O_{\left(T_{i}, k\right)}$ keeps both keys $k_{i}^{\text {old }}=k_{(i, j)}$ and $k_{i}^{\text {new }}=$ $G\left(k_{(i, j)}, N\right), O_{\left(T_{i}, k\right)}$ can always re-synchronize with $T_{i}$ using $k_{i}^{\text {old }}$.

In the rest of this section, we assume that the channels between the owners are secure.

Issuer verification protocol. In order to verify whether a tag $T_{i}$ owned by $O_{\left(T_{i}, k\right)}$ is actually issued by $\mathcal{I}$, a verifier $\mathcal{V}$ proceeds as follows:

1. $\mathcal{V}$ sends a nonce $N$ to $T_{i}$, as depicted in Figure 3 .

Upon receiving $N, T_{i}$ replies with $c_{(i, j)}=\left(u_{(i, j)}, v_{(i, j)}\right)=\left(g_{1}^{r_{(i, j)}}, h_{i}^{x} g_{1}^{\alpha_{k}^{2} r_{(i, j)}}\right)$, a random number $R_{(i, j)}$, and $\sigma_{(i, j)}=\operatorname{HMAC}_{k_{(i, j)}}\left(N, R_{(i, j)}, c_{(i, j)}\right)$.

2. Once $\mathcal{V}$ receives $T_{i}$ 's reply, he chooses a random number $r_{v} \in \mathbb{F}_{q}^{*}$ and computes $A_{v}=\left(u_{(i, j)}\right)^{r_{v}}=g_{1}^{r_{(i, j)} r_{v}}$.

3. Then, $\mathcal{V}$ sends $N, R_{(i, j)}, c_{(i, j)}, \sigma_{(i, j)}$ along with $A_{v}$ to $O_{\left(T_{i}, k\right)}$. Note that $r_{v}$ and therewith $A_{v}$ is used to prevent replay attacks by $O_{\left(T_{i}, k\right)}$.

When receiving the tuple $\left(N, R_{(i, j)}, c_{(i, j)}, \sigma_{(i, j)}, A_{v}\right), O_{\left(T_{i}, k\right)}$ identifies and authenticates $T_{i}$. If $O_{\left(T_{i}, k\right)}$ is not willing to run the verification protocol for $T_{i}$ he aborts the verification. Otherwise, $O_{\left(T_{i}, k\right)}$ computes: $\operatorname{ref}_{T_{i}}^{V}=\left(A_{(i, j)}, B_{(i, j)}\right.$, $\left.C_{(i, j)}\right)=\left(t_{i}, H\left(t_{i}\right)^{x}, A_{v}^{\alpha_{k}}\right)$.

4. $O_{\left(T_{i}, k\right)}$ sends $\operatorname{ref}_{T_{i}}^{V}=\left(A_{(i, j)}, B_{(i, j)}, C_{(i, j)}\right)$ to $\mathcal{V}$.

Given the verification references $\operatorname{ref}_{T_{i}}^{V}, \mathcal{V}$ checks whether the following equations hold:

$$
\begin{gathered}
e\left(H\left(A_{(i, j)}\right), g_{2}^{x}\right)=e\left(B_{(i, j)}, g_{2}\right) \\
e\left(C_{(i, j)}, g_{2}\right)=e\left(A_{v}, g_{2}^{\alpha_{k}}\right)
\end{gathered}
$$

Equation (1) verifies whether $B_{(i, j)}=H\left(A_{(i, j)}\right)^{x}$, i.e., whether $B_{(i, j)}$ is the signature of $A_{(i, j)}$ by issuer $\mathcal{I}$. Equation (2) checks whether $C_{(i, j)}=A_{v}^{\alpha_{k}}$.

Finally, $\mathcal{V}$ verifies whether $c_{(i, j)}$ is the encryption of $B_{(i, j)}$ with the public key $g_{1}^{\alpha_{k}^{2}}$ by checking if the following equation holds:

$$
e\left(v_{(i, j)}, g_{2}\right)^{r_{v}}=e\left(B_{(i, j)}, g_{2}\right)^{r_{v}} e\left(C_{(i, j)}, g_{2}^{\alpha_{k}}\right)
$$




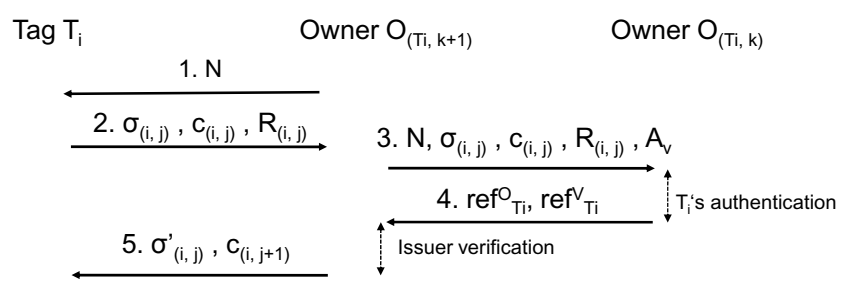

Fig. 4. Ownership transfer in ROTIV

Note that if $c_{(i, j)}$ is the encryption of $B_{(i, j)}$ with the public key $g_{1}^{\alpha_{k}^{2}}$, we have:

$$
\begin{aligned}
& c_{(i, j)}=\left(u_{(i, j)}, v_{(i, j)}\right)=\left(g_{1}^{r_{(i, j)}}, B_{(i, j)} g_{1}^{\alpha_{k}^{2} r_{(i, j)}}\right) \text {. Therefore, } \\
& \begin{aligned}
e\left(v_{(i, j)}, g_{2}\right)^{r_{v}} & =e\left(B_{(i, j)}, g_{2}\right)^{r_{v}} e\left(g_{1}^{\alpha_{k}^{2} r_{(i, j)}}, g_{2}\right)^{r_{v}}=e\left(B_{(i, j)}, g_{2}\right)^{r_{v}} e\left(g_{1}^{r_{v} r_{(i, j)}}, g_{2}^{\alpha_{k}^{2}}\right) \\
& =e\left(B_{(i, j)}, g_{2}\right)^{r_{v}} e\left(A_{v}, g_{2}^{\alpha_{k}^{2}}\right)=e\left(B_{(i, j)}, g_{2}\right)^{r_{v}} e\left(A_{v}^{\alpha_{k}}, g_{2}^{\alpha_{k}}\right) \\
& =e\left(B_{(i, j)}, g_{2}\right)^{r_{v}} e\left(C_{(i, j)}, g_{2}^{\alpha_{k}}\right)
\end{aligned}
\end{aligned}
$$

If all the equations hold, $\mathcal{V}$ outputs $b=1$ meaning that $\mathcal{I}$ is $T_{i}$ 's issuer. Otherwise, $\mathcal{V}$ outputs $b=0$ meaning that $\mathcal{I}$ is not the issuer of $T_{i}$.

Ownership transfer protocol. The setup of the ownership transfer in ROTIV consists of a current owner $O_{\left(T_{i}, k\right)}$, a prospective owner $O_{\left(T_{i}, k+1\right)}$ and a tag $T_{i}$ as shown in Figure 4. The ownership transfer consists of: a) a mutual authentication between $T_{i}$ and $\left.O_{\left(T_{i}, k+1\right)}, \mathbf{b}\right)$ an exchange of verification references $\operatorname{ref}_{T_{i}}^{V}$ between $O_{\left(T_{i}, k\right)}$ and $O_{\left(T_{i}, k+1\right)}$ to perform issuer verification, and c) an exchange of ownership references $\operatorname{ref}_{T_{i}}^{O}$ between $O_{\left(T_{i}, k\right)}$ and $O_{\left(T_{i}, k+1\right)}$ to allow $O_{\left(T_{i}, k+1\right)}$ authentication.

The ownership transfer protocol between $O_{\left(T_{i}, k\right)}$ and $O_{\left(T_{i}, k+1\right)}$ for tag $T_{i}$ is as follows:

1. The owner $O_{\left(T_{i}, k+1\right)}$ sends a nonce $N$ to $\operatorname{tag} T_{i}$.

2. $T_{i}$ replies with $c_{(i, j)}=\left(u_{(i, j)}, v_{(i, j)}\right)$, a random number $R_{(i, j)}$ and HMAC $\sigma_{(i, j)}$

3. $O_{\left(T_{i}, k+1\right)}$ selects a random number $r_{v}$ and computes $A_{v}=u_{(i, j)}^{r_{v}}$. $O_{\left(T_{i}, k+1\right)}$ sends $N, R_{(i, j)}, c_{(i, j)}, \sigma_{(i, j)}$ and $A_{v}$ to $T_{i}$ 's owner $O_{\left(T_{i}, k\right)}$.

Given $N, R_{(i, j)}, c_{(i, j)}$ and $\sigma_{(i, j)}, O_{\left(T_{i}, k\right)}$ authenticates $T_{i}$. If the authentication fails, $O_{\left(T_{i}, k\right)}$ informs $O_{\left(T_{i}, k+1\right)}$, who re-sends his first message to $T_{i}$. Otherwise, $O_{\left(T_{i}, k\right)}$ supplies $O_{\left(T_{i}, k+1\right)}$ with: $\operatorname{ref}_{T_{i}}^{O}=\left(k_{i}^{\text {old }}, k_{i}^{\text {new }}, x_{i}, y_{i}\right)=$ $\left(k_{(i, j)}, k_{(i, j)}, t_{i}, h_{i}^{x}=H\left(t_{i}\right)^{x}\right)$ and $\operatorname{ref}_{T_{i}}^{V}=\left(A_{(i, j)}, B_{(i, j)}, C_{(i, j)}\right)=\left(t_{i}, h_{i}^{x}, A_{v}^{\alpha_{k}}\right)$.

4. Provided with $\operatorname{ref}_{T_{i}}^{O}, O_{\left(T_{i}, k+1\right)}$ checks if the equation $\sigma_{(i, j)}=\operatorname{HMAC}_{k_{(i, j)}}(N$, $\left.R_{(i, j)}, c_{(i, j)}\right)$ holds. If it does, this implies that the key $k_{(i, j)}$ provided by $O_{\left(T_{i}, k\right)}$ corresponds to tag $T_{i}$.

Given $\operatorname{ref}_{T_{i}}^{V}, O_{\left(T_{i}, k+1\right)}$ verifies whether the issuer of $T_{i}$ is $\mathcal{I}$. If the verification fails, $O_{\left(T_{i}, k+1\right)}$ aborts the ownership transfer. If not, $O_{\left(T_{i}, k+1\right)}$ adds the entry $\left(y_{i}, \operatorname{ref}_{T_{i}}^{O}\right)$ into his database $\mathcal{D}_{k+1}$, and finishes the authentication with $T_{i}$. $O_{\left(T_{i}, k+1\right)}$ chooses a new random number $r_{(i, j+1)} \in \mathbb{F}_{q}^{*}$ and computes: 


$$
\begin{aligned}
c_{(i, j+1)} & =\left(u_{(i, j+1)}, v_{(i, j+1)}\right)=\left(g_{1}^{r_{(i, j+1)}}, y_{i} g_{1}^{\alpha_{k+1}^{2} r_{(i, j+1)}}\right) \\
\sigma_{(i, j)}^{\prime} & =\operatorname{HMAC}_{k_{(i, j)}}\left(R_{(i, j)}, c_{(i, j+1)}\right)
\end{aligned}
$$

So, $c_{(i, j+1)}$ is the encryption of $y_{i}$ with $O_{\left(T_{i}, k+1\right)}$ 's public key $g_{1}^{\alpha_{k+1}^{2}}$.

5. $O_{\left(T_{i}, k+1\right)}$ sends $c_{(i, j+1)}$ and $\sigma_{(i, j)}^{\prime}$ to $T_{i}$, and updates its database $\mathcal{D}_{k+1}$ as in the authentication protocol presented above.

Upon receiving $c_{(i, j+1)}$ and $\sigma_{(i, j)}^{\prime}, T_{i}$ authenticates $O_{\left(T_{i}, k+1\right)}$. If the authentication succeeds $T_{i}$ updates its state accordingly.

Note. To prevent the old owner $O_{\left(T_{i}, k\right)}$ from tracing the tag later in the future, the new owner $O_{\left(T_{i}, k+1\right)}$ has to run a mutual authentication with $T_{i}$ outside the range of $O_{\left(T_{i}, k\right)}$ after the ownership transfer. In this manner, $T_{i}$ and $O_{\left(T_{i}, k+1\right)}$ will share a symmetric key that $O_{\left(T_{i}, k\right)}$ cannot retrieve without physical access to tag $T_{i}$.

\section{$5 \quad$ Privacy and Security Models}

We assume that the communication channel between owners during an ownership transfer and an owner and a verifier during an issuer verification protocol are secure. That is, an adversary $\mathcal{A}$ has only access to the interactions between tags and owners and the wireless interactions between tags and verifiers.

\subsection{Privacy}

Inspired by previous work on ownership transfer [13, 5], we formally define using experiments the two major privacy requirements for ownership transfer which are tag forward unlinkability and tag backward unlinkability. In the setting of tag ownership transfer, forward unlinkability ensures that when a new owner $O_{(T, k+1)}$ acquires $T$ 's secrets after a successful ownership transfer at time $k+1$, he still cannot tell whether $T$ has participated in protocol runs at time $t<k+1$. On the other hand, backward unlinkability, ensures that when a previous owner $O_{(T, k)}$ releases tag's ownership at time $k+1$, he still cannot tell whether $T$ is involved in interactions that occured at time $t>k+1$.

In the remainder of this section, we assume that the adversary $\mathcal{A}$ has access to oracles:

- $\mathcal{O}_{\mathcal{T}}$ is an oracle that, when queried, randomly returns a tag $T$ from the set of tags $\mathcal{T}$.

- $\mathcal{O}_{\text {flip }}$ is an oracle that, when queried with two tags $T_{0}$ and $T_{1}$, randomly chooses $b \in\{0,1\}$ and returns $T_{b}$.

$-\mathcal{O}_{\mathbb{O}}$ is an oracle that, when queried, returns a randomly selected owner $O$ from the set of legitimate owners $\mathbb{O}$.

Forward unlinkability. The forward unlinkability experiment captures the capabilities of adversary $\mathcal{A}$ who is allowed to own a tag $T$ at the end of his attack, and who has to decide if $T$ was already involved in previous interactions. 
Note that in scenarios where mutual authentication is required, the notion of forward unlinkability has been proven to be unachievable without tag performing public key cryptography operations, see Paise and Vaudenay [16].

Thus, as discussed in Section 3, in order to achieve constant time authentication and denial of service resistance, we assume that there is at least one communication between $T$ and its owner that is un-observed by $\mathcal{A}$.

Our forward unlinkability experiment is indistinguishability based as proposed by Juels and Weis [9]. Adversary $\mathcal{A}(r, s, t, \epsilon)$ has access to tags in two phases. In the learning phase, as depicted in Algorithm 1, oracle $\mathcal{O}_{\mathcal{T}}$ gives $\mathcal{A}$ two tags $T_{0}$ and $T_{1}$ that he can eavesdrop on by calling OBSERvEINTERACTION $\left(T_{i}\right)$ for a maximum of $t$ times. Note that OBSERVEINTERACTION $\left(T_{i}\right)$ eavesdrops on tag $T_{i}$ during mutual authentications, ownership transfer or issuer verification.

In addition to $T_{0}$ and $T_{1}, \mathcal{O}_{\mathcal{T}}$ gives $\mathcal{A}$ a set of $r$ tags $T_{i}^{\prime}$. The ownership of $T_{i}^{\prime}$ is then transferred to $\mathcal{A}$ through Transfer Ownership $\left(T_{i}^{\prime}, O_{\left(T_{i}^{\prime}, k\right)}, \mathcal{A}\right)$. $\mathcal{A}$ is now allowed to run up to $s$ mutual authentication with $T_{i}^{\prime}$.

In the challenge phase as depicted in Algorithm 2, $T_{0}$ and $T_{1}$ run once a mutual authentication with their respective owners (cf., RunAUTH) outside the range of the adversary $\mathcal{A}$. Then, the oracle $\mathcal{O}_{\text {flip }}$ queried with the tags $T_{0}$ and $T_{1}$, selects randomly $b \in\{0,1\}$ and returns the $\operatorname{tag} T_{b}$ to $\mathcal{A}$. Then, the ownership of $\operatorname{tag} T_{b}$ will be transferred to $\mathcal{A}$. Then, $\mathcal{A}$ can run up to $t$ mutual authentication with $\operatorname{tag} T_{b}$.

$\mathcal{A}$ calls as well oracle $\mathcal{O}_{\mathcal{T}}$ that supplies him with $r$ tags $T_{i}^{\prime \prime}$. Then, the ownership of $T_{i}^{\prime \prime}$ is transferred to $\mathcal{A}$, who now can run up to $s$ mutual authentication with $T_{i}^{\prime \prime}$. Finally, $\mathcal{A}$ outputs his guess of the value of $b$.

$\mathcal{A}$ is successful, if his guess of $b$ is correct.

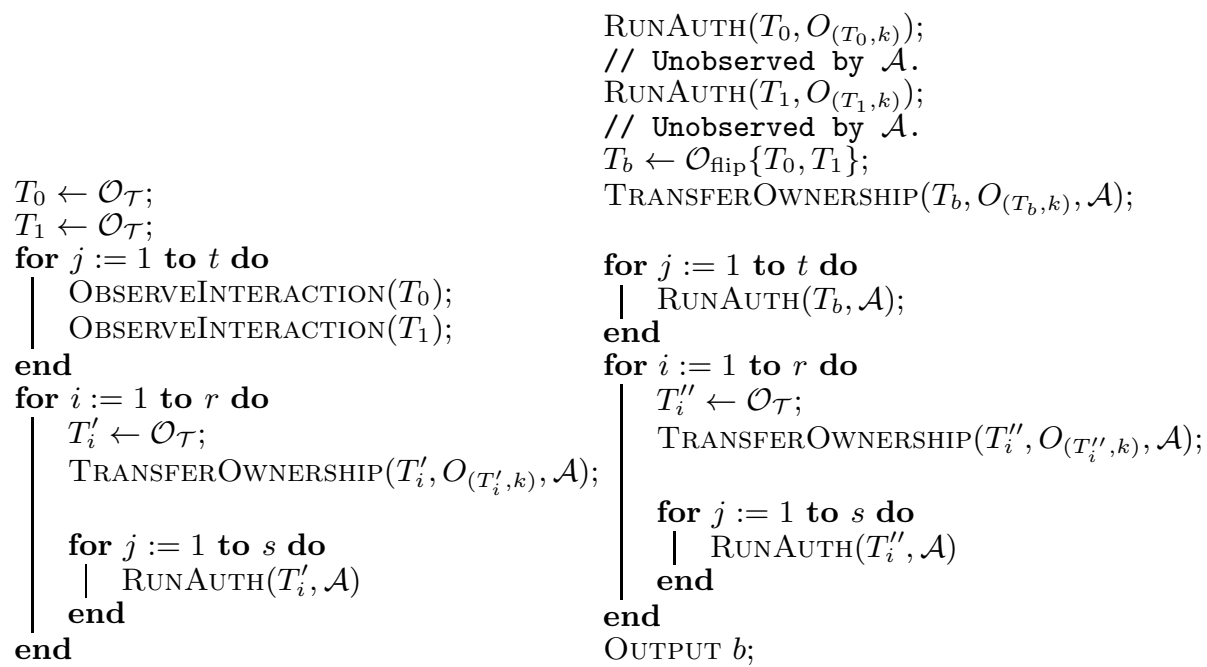

Algorithm 1. A's forward unlinkability learning phase

Algorithm 2. A's forward unlinkability challenge phase 
Definition 3 (Forward Unlinkability). ROTIV provides forward unlinkability $\Leftrightarrow$ For any adversary $\mathcal{A}$, inequality $\operatorname{Pr}(\mathcal{A}$ is successful $) \leq \frac{1}{2}+\epsilon$ holds, where $\epsilon$ is negligible.

Backward unlinkability. It has been shown in [19] that is impossible to achieve backward unlinkability without public key cryptography on tags. In order to achieve at least a slightly weaker notion of backward unlinkability, we add the assumption that a previous owner $O_{(T, k)}$ of $\operatorname{tag} T$ cannot continuously monitor $T$ after releasing $T$ 's ownership. This has been previously suggested by, e.g., Lim and Kwon [13], Dimitrou [5].

The backward unlinkability experiment captures the capabilities of an adversary $\mathcal{A}$ who releases the ownership of tag $T$ during his attack and has to tell whether $T$ is involved in future protocol transactions.

In the learning phase, cf., Algorithm 3, oracle $\mathcal{O}_{\mathcal{T}}$ selects randomly two tags $T_{0}$ and $T_{1}$. Then, the ownership of these two tags is transferred to $\mathcal{A}$. $\mathcal{A}$ is allowed to run up to $t$ mutual authentications with tags $T_{0}$ and $T_{1}$.

$\mathcal{O}_{\mathcal{T}}$ gives $\mathcal{A}$ also a set of $r$ tags $T_{i}^{\prime}$. Then, the ownership of tags $T_{i}^{\prime}$ is transferred to $\mathcal{A}$, who can then perform up to $s$ mutual authentications with tags $T_{i}^{\prime}$.

At the end of the learning phase, the oracle $\mathcal{O}_{\mathbb{O}}$ supplies $\mathcal{A}$ with two randomly selected owners. $\mathcal{A}$ then, releases the ownership of tags $T_{0}$ and $T_{1}$.

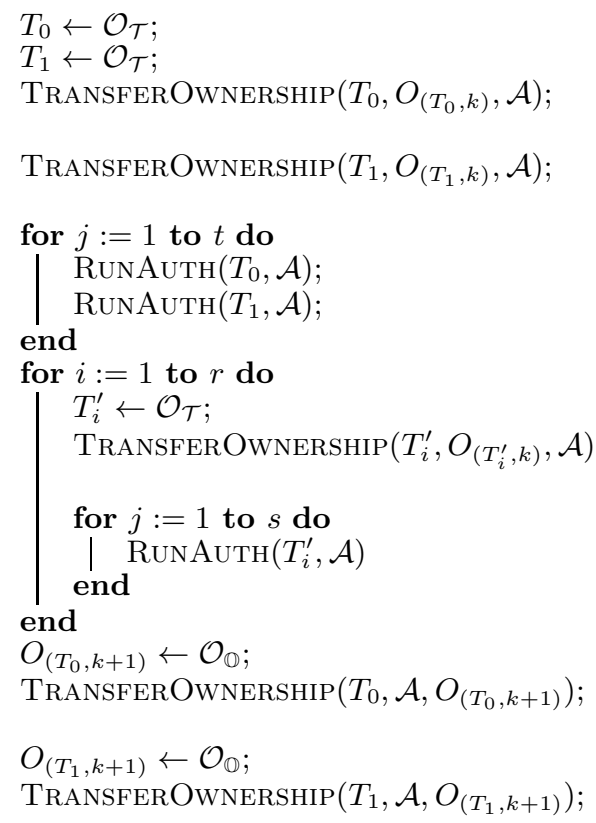

Algorithm 3. $\mathcal{A}$ 's backward unlinkability learning phase

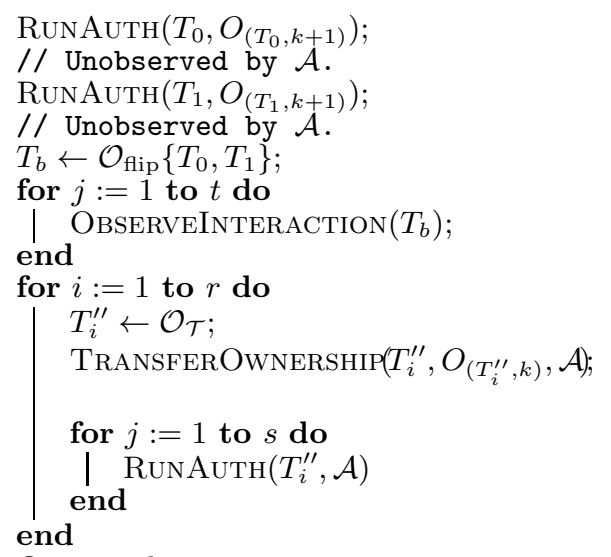

Output $b$;

Algorithm 4. $\mathcal{A}$ 's backward unlinkability challenge phase

In the challenge phase as depicted in Algorithm $4 . T_{0}$ and $T_{1}$ run a mutual authentication with their respective owners outside the range of the adversary 
$\mathcal{A}$. The oracle $\mathcal{O}_{\text {flip }}$ queried with tags $T_{0}$ and $T_{1}$, chooses randomly $b \in\{0,1\}$ and returns the tag $T_{b}$ to $\mathcal{A}$. $\mathcal{A}$ is allowed to eavesdrop on $T_{b}$ for a maximum of $t$ times.

$\mathcal{A}$ queries also the oracle $\mathcal{O}_{\mathcal{T}}$ that supplies $\mathcal{A}$ with $r$ tags $T_{i}^{\prime \prime}$. The ownership of $T_{i}^{\prime \prime}$ is transferred to $\mathcal{A}$, who is allowed to run up to $s$ mutual authentication with $T_{i}^{\prime \prime}$. Finally, $\mathcal{A}$ outputs his guess of the value of $b . \mathcal{A}$ is successful, if his guess of $b$ is correct.

Definition 4 (Backward Unlinkability). ROTIV provides backward unlinkability $\Leftrightarrow$ For any adversary $\mathcal{A}$, inequality $\operatorname{Pr}(\mathcal{A}$ is successful $) \leq \frac{1}{2}+\epsilon$ holds, where $\epsilon$ is negligible.

\subsection{Security}

As ROTIV consists of two main protocols, an ownership transfer protocol and an issuer verification protocol, we introduce the security requirements for each protocol separately. The adversary $\mathcal{A}$ in this section is a direct adaptation of the non-narrow destructive adversary by Vaudenay [19] and Paise and Vaudenay [16] to tag ownership transfer in supply chains.

Ownership transfer. A secure ownership transfer must assure the following properties:

a) Mutual authentication. A secure ownership transfer protocol must ensure that, when a $\operatorname{tag} T$ runs a successful mutual authentication with owner $O$, this implies that $O$ is $T$ 's current owner with high probability. Also, when an owner $O$ runs a successful mutual authentication with a $\operatorname{tag} T$, it yields that $T$ is a legitimate tag with high probability.

We define an authentication game in accordance with Lim and Kwon 13], Vaudenay [19] and Paise and Vaudenay [16]. This game proceeds in two phases. During the learning phase as depicted in Algorithm [5, an adversary $\mathcal{A}(r, s, t, \epsilon)$ is supplied with a challenge tag $T_{c}$ from oracle $\mathcal{O}_{\mathcal{T}}$. $\mathcal{A}$ is not allowed to read the internal state of $T_{c} . \mathcal{A}$ is allowed to eavesdrop on $r$ mutual authentications between $T_{c}$ and its owner $O_{\left(T_{c}, k\right)}$, cf., RunAuth $\left(T_{c}, O_{\left(T_{c}, k\right)}\right)$. He can also alter authentications by modifying the messages exchanged between $T_{c}$ and its owner $O_{\left(T_{c}, k\right)}$, cf., AlterAuth $\left(T_{c}, O_{\left(T_{c}, k\right)}\right) . \mathcal{A}$ is allowed as well to start $s$ authentications with $T_{c}$ while impersonating $O_{\left(T_{c}, k\right)},\left(\mathrm{cf}\right.$., $\operatorname{RunAuth}\left(T_{c}, \mathcal{A}\right)$ ). Also he can start $t$ authentications with $O_{\left(T_{c}, k\right)}$ while impersonating $T_{c}$, cf., $\operatorname{RunAuth}(\mathcal{A}$, $\left.O_{\left(T_{c}, k\right)}\right)$.

$\mathcal{A}$ 's goal in the challenge phase is either to run a successful mutual authentication with $T_{c}$, i.e., $\mathcal{A}$ succeeds in impersonating $O_{\left(T_{c}, k\right)}$, or to run a successful mutual authentication with $O_{\left(T_{c}, k\right)}$, i.e., $\mathcal{A}$ succeeds in impersonating $T_{c}$.

In the challenge phase as depicted in Algorithm 6, $\mathcal{A}(r, s, t, \epsilon)$ interacts with $T_{c}$ and initiates an authentication protocol run to impersonate $O_{\left(T_{c}, k\right)}$, cf., $\operatorname{RunAuth}\left(T_{c}, \mathcal{A}\right)$. At the end of the authentication, $T_{c}$ outputs a bit $b_{T_{c}}, b_{T_{c}}=1$ if the authentication with $\mathcal{A}$ was successful, and $b_{T_{c}}=0$ otherwise. 


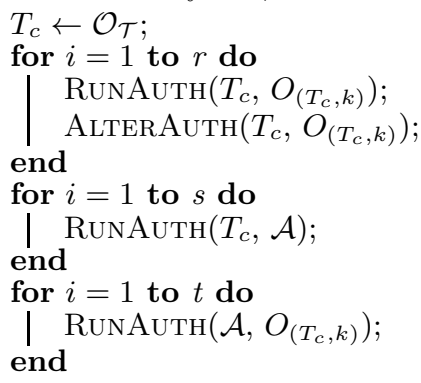

Algorithm 5. $\mathcal{A}$ 's authentication learning phase

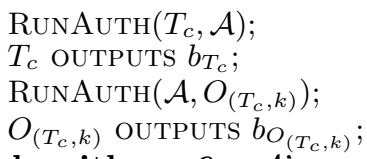

Algorithm 6. $\mathcal{A}$ 's authentication challenge phase

$\mathcal{A}$ can interact as well with $O_{\left(T_{c}, k\right)}$ and initiates an authentication protocol run to impersonate $T_{c}$, cf., RunAuth $\left(\mathcal{A}, O_{\left(T_{c}, k\right)}\right)$. At the end of this authentication, $O_{\left(T_{c}, k\right)}$ outputs a bit $b_{O_{\left(T_{c}, k\right)}}=1$, if the authentication was successful, $b_{O_{\left(T_{c}, k\right)}}=$ 0 otherwise.

$\mathcal{A}$ is successful if, $b_{T_{c}}=1$ or $b_{O_{\left(T_{c}, k\right)}}=1$.

Definition 5 (Authentication). ROTIV is secure with regard to authentication $\Leftrightarrow$ For any adversary $\mathcal{A}$, inequality $\operatorname{Pr}(\mathcal{A}$ is successful $) \leq \epsilon$ holds, where $\epsilon$ is negligible.

b) Exclusive ownership It ensures that an adversary $\mathcal{A}$ who does not have $T$ 's ownership references noted $\operatorname{ref}_{T}^{O}$, cannot transfer the ownership of $T$, unless he rewrites the content of $T$.

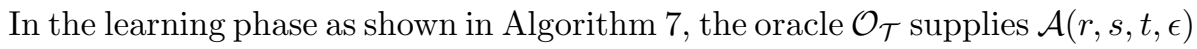
with $r \operatorname{tags} T_{i}$, then, the ownership of $\operatorname{tag} T_{i}$ is transferred to $\mathcal{A}$. $\mathcal{A}$ can run up to $s$ successful mutual authentications with $T_{i}$, cf., $\operatorname{RunAuth}\left(T_{i}, \mathcal{A}\right)$. He can as well at the end of the learning phase, transfer the ownership of tag $T_{i}$ to an owner $O_{i}$ selected randomly from the set of owners $\mathbb{O}$.

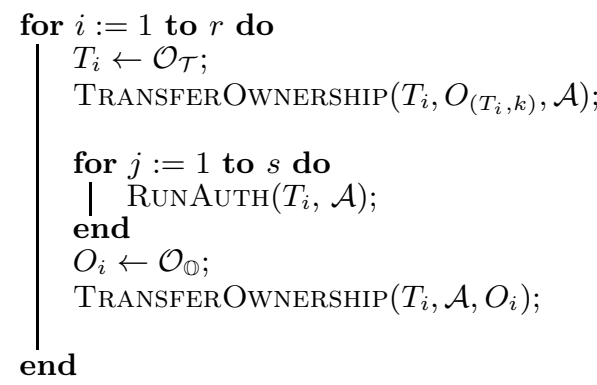

Algorithm 7. $\mathcal{A}$ 's exclusive ownership learning phase

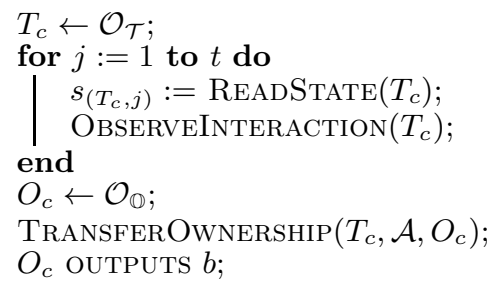

Algorithm 8. $\mathcal{A}$ 's exclusive ownership challenge phase

In the challenge phase, cf., Algorithm 8 the oracle $\mathcal{O}_{\mathcal{T}}$ gives $\mathcal{A}(r, s, t, \epsilon)$ a challenge tag $T_{c}$.

$\mathcal{A}$ can read $T_{c}$ 's internal state, cf., ReadState $\left(T_{c}\right)$, and eavesdrop on $T_{c}$ 's up to $t$ times. However, $\mathcal{A}$ is not allowed to alter $T_{c}$ 's internal state. At the end of the challenge phase, $\mathcal{A}$ queries the oracle $\mathcal{O}_{\mathbb{O}}$. $\mathcal{O}_{\mathbb{O}}$ returns a challenge owner $O_{c}$. 
$\mathcal{A}$ runs an ownership transfer protocol for $T_{c}$ with $O_{c} . O_{c}$ outputs a bit $b=1$, if the ownership transfer was successful, and $b=0$ otherwise. $\mathcal{A}$ is successful, if $b=1$.

Definition 6 (Exclusive ownership). ROTIV provides exclusive ownership $\Leftrightarrow$ For any adversary $\mathcal{A}$, inequality $\operatorname{Pr}(\mathcal{A}$ is successful $) \leq \epsilon$ holds, where $\epsilon$ is negligible.

Issuer verification The security of issuer verification ensures that when a verifier $\mathcal{V}$ outputs that the issuer of $\operatorname{tag} T$ is $\mathcal{I}$, it implies that $\mathcal{I}$ is the issuer of $T$ with high probability.

An adversary $\mathcal{A}$ 's goal is to run an issuer verification protocol with $\mathcal{V}$ for tag $T$ that was not issued by $\mathcal{I}$, and still $\mathcal{V}$ outputs that $\mathcal{I}$ is the issuer of $T$.

In the learning phase, $\mathcal{A}$ queries the oracle $\mathcal{O}_{\mathcal{T}}$ that gives $\mathcal{A}$ a total of $r$ random tags $T_{i}$. The ownership of $T_{i}$ is then transferred to $\mathcal{A}$, cf. TransferOwner$\operatorname{SHIP}\left(O_{\left(T_{i}, k\right)}, \mathcal{A}, T_{i}\right) . \mathcal{A}$ can run up to $s$ mutual authentications with $\operatorname{tag} T_{i}$, cf., $\operatorname{RunAuth}\left(T_{c}, \mathcal{A}\right)$. The adversary can also run $s$ issuer verification protocol for $\operatorname{tag} T_{i}$ with the verifier $\mathcal{V}$, cf., $\operatorname{VerIFy}\left(T_{i}, \mathcal{A}, \mathcal{V}\right)$ and to transfer $T_{i}$ 's ownership to an owner $O_{i}$ randomly selected from the set of owners $\mathbb{O}$.

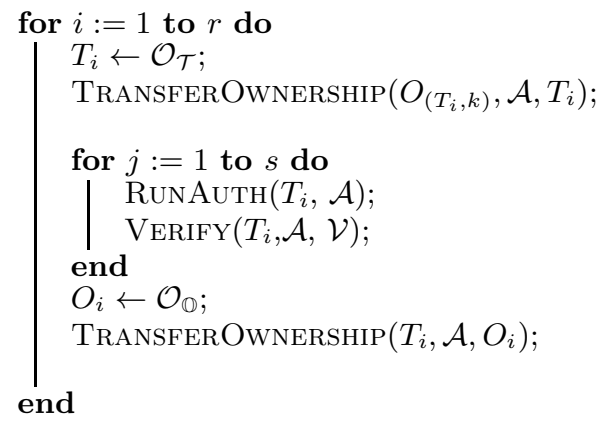

CreateTag $T_{c}$; $\operatorname{MODifySTATE}\left(T_{c}, s_{T_{c}}^{\prime}\right)$ $\operatorname{VERIFY}\left(T_{c}, \mathcal{A}, \mathcal{V}\right)$ $\mathcal{V}$ OUTPUTS $b$;

Algorithm 10. $\mathcal{A}$ 's issuer verification security challenge phase Algorithm 9. $\mathcal{A}$ 's issuer
security learning phase

In the challenge phase, $\mathcal{A}$ creates a tag $T_{c} \notin \mathcal{T}$ and write some state $s_{T_{c}}^{\prime}$ in it. Then, $\mathcal{A}$ starts a verification protocol for $\operatorname{tag} T_{c}$ with the verifier $\mathcal{V}$, cf., $\operatorname{VERIFY}\left(T_{c}, \mathcal{A}, \mathcal{V}\right)$. Finally, $\mathcal{V}$ outputs a bit $b=1$, if the issuer verification protocol outputs $\mathcal{I}$, and $b=0$ otherwise. $\mathcal{A}$ is successful, if $b=1$ and $s_{T_{c}}^{\prime}$ does not correspond to a state of tag $T_{i}$ that was given to $\mathcal{A}$ in the learning phase.

Definition 7 (Issuer verification security). ROTIV is secure with regard to issuer verification $\Leftrightarrow$ For any adversary $\mathcal{A}$, inequality $\operatorname{Pr}(\mathcal{A}$ is successful $) \leq \epsilon$ holds, where $\epsilon$ is negligible.

\section{Privacy and Security Analysis}

In this section, we state the main proofs of ROTIV's privacy and security.

Due to space limitations, we give only proof sketches. The detailed proofs could be found in the extended version of this paper [6]. 


\subsection{Privacy}

We prove that ROTIV provides forward unlinkability and backward unlinkability under the SXDH assumption (DDH is hard in both $\mathbb{G}_{1}$ and $\mathbb{G}_{2}$ ).

Theorem 1 (Forward unlinkability). ROTIV provides forward unlinkability under the $S X D H$ assumption.

Proof (sketch). Assume there is an adversary $\mathcal{A}$ who breaks the forward unlinkability ROTIV with a non negligible advantage $\epsilon$. We show that there is an adversary $\mathcal{A}^{\prime}$ that uses $\mathcal{A}$ to break the DDH assumption, that is, given $\left(g_{1}, g_{1}^{\alpha}, g_{1}^{\beta}, g_{1}^{\gamma}\right)$, it is hard to decide whether $\gamma=\alpha \beta$.

To break DDH, $\mathcal{A}^{\prime}$ creates a ROTIV system with 1.) issuer $\mathcal{I}$ whose public key is $g_{2}^{x}$ where $x$ is selected randomly from $\mathbb{F}_{q}$. 2.) Owner $O$ whose public key is $g_{1}^{\alpha}$.

To issue the challenge tags $T_{i}, i \in\{0,1\}, \mathcal{A}^{\prime}$ randomly selects $t_{i}, k_{(i, 0)}$ and $r_{(i, 0)} \in \mathbb{F}_{q}$, then computes $h_{i}=H\left(t_{i}\right)$, and $c_{(i, 0)}=\left(u_{(i, 0)}, v_{(i, 0)}\right)=\left(g_{1}^{\beta r_{(i, 0)}}, h_{i}^{x}\right.$ $\left.g_{1}^{\gamma r(i, 0)}\right)$. Finally, $\mathcal{A}^{\prime}$ stores $s_{(i, 0)}=\left(k_{(i, 0)}, c_{(i, 0)}\right)$ in tag $T_{i}$.

In the challenge phase, $\mathcal{A}^{\prime}$ starts authentications outside the range of $\mathcal{A}$ with $T_{0}$ by sending a nonce $N_{0}$ and with $T_{1}$ by sending a nonce $N_{1}$. We assume $T_{0}$ stores $s_{(0, j)}=\left(k_{(0, j)}, c_{(0, j)}\right)$ and $T_{1}$ stores $s_{(1, j)}=\left(k_{(1, j)}, c_{(1, j)}\right)$.

At the end of an authentication, $\mathcal{A}^{\prime}$ updates the state of $T_{0}$ and $T_{1}$ as follows: $s_{(i, j+1)}=\left(k_{(i, j+1)}, c_{(i, j+1)}\right), i \in\{0,1\}$, where $k_{(i, j+1)}=G\left(N_{i}, k_{(i, j)}\right)$ and $c_{(i, j+1)}=\left(g_{1}^{r_{(i, j+1)}}, h_{i}^{x} g_{1}^{\alpha r_{(i, j+1)}}\right)$.

Given that $\mathcal{A}$ does not have access to $N_{i}, i \in\{0,1\}, k_{(i, j+1)}=G\left(k_{(i, j)}, N_{i}\right)$ cannot give $\mathcal{A}$ any information about $T_{b}$ 's past interactions. So, the privacy of ROTIV is reduced to the security of ciphertexts stored in $T_{0}$ and $T_{1}$.

In the challenge phase, $\mathcal{A}^{\prime}$ selects randomly a coin $b \in\{0,1\}$ and transfers the ownership of $T_{b}$ to $\mathcal{A}$. $T_{b}$ now stores a state $s_{(b, j+1)}=\left(k_{(b, j+1)}, c_{(b, j+1)}\right)$ where $c_{(b, j+1)}=\left(g_{1}^{r_{(b, j+1)}}, h_{b}^{x} g_{1}^{\alpha r_{(b, j+1)}}\right)$.

At the end of the challenge phase, $\mathcal{A}$ outputs his guess of $b$.

If $\gamma=\alpha \beta$, then $c_{(b, j+1)}$ is re-encryption of $c_{(b, j)}$. $\mathcal{A}$ then can output a correct guess for the tag corresponding to $T_{b}$ with a non-negligible advantage $\epsilon$. Thus, $\mathcal{A}^{\prime}$ can tell that $\gamma=\alpha \beta$ with a non negligible advantage $\epsilon$. $\frac{1}{2}$.

If $\gamma \neq \alpha \beta$, the probability that $\mathcal{A}^{\prime}$ can break the DDH is a random guess, i.e.,

Let $E_{1}$ be the event that $\mathcal{A}^{\prime}$ can break DDH, and $E_{2}$ is the event that $\gamma=\alpha \beta$ holds. The probability of event $E_{2}$ is $\frac{1}{2}$.

$$
\begin{aligned}
\operatorname{Pr}\left(E_{1}\right) & =\operatorname{Pr}\left(E_{2}\right) \cdot \operatorname{Pr}\left(E_{1} \mid E_{2}\right)+\operatorname{Pr}\left(\overline{E_{2}}\right) \cdot \operatorname{Pr}\left(E_{1} \mid \overline{E_{2}}\right) \\
& =\frac{1}{2} \operatorname{Pr}\left(E_{1} \mid E_{2}\right)+\frac{1}{2} \operatorname{Pr}\left(E_{1} \mid \overline{E_{2}}\right) \geq \frac{1}{2}\left(\frac{1}{2}+\epsilon\right)+\frac{1}{2} \cdot \frac{1}{2}=\frac{1}{2}+\frac{\epsilon}{2}
\end{aligned}
$$

Therefore, with $\mathcal{A}$ 's non negligible advantage in breaking forward unlinkability of ROTIV, $\mathcal{A}^{\prime}$ 's advantage in breaking DDH in $\mathbb{G}_{1}$ is also non negligible.

Theorem 2 (Backward unlinkability). ROTIV provides backward unlinkability under the SXDH assumption. 
The same reasoning of the above proof sketch applies for ROTIV's backward unlinkability.

\subsection{Security}

We prove that ROTIV is secure with regards to the security properties introduced in Section 5.2 .

Theorem 3 (Secure authentication). The ownership transfer protocol in ROTIV provides secure authentication under the security of HMAC.

Proof (sketch). Assume there is an adversary $\mathcal{A}$ who breaks the mutual authentication of ROTIV with a non-negligible advantage. We show that there is an adversary $\mathcal{A}^{\prime}$ who breaks the security of HMAC with a non-negligible advantage.

Note that if $\mathcal{A}$ breaks the mutual authentication of ROTIV, then this means that $\mathcal{A}$ is able to either impersonate the challenge tag $T_{c}$ or to impersonate the owner of $T_{c}$.

Let $k$ denotes the secret key shared between $T_{c}$ and $T_{c}$ 's owner in the challenge phase of the mutual authentication experiment.

1) If $\mathcal{A}$ impersonates $T_{c}$ : this implies that $\mathcal{A}$ receives a nonce $N$ from $T_{c}$ 's owner and then replies with a ciphertext $c$, a random number $R$ and $\sigma=$ $\operatorname{HMAC}_{k}(N, R, c)$.

An adversary $\mathcal{A}^{\prime}$ who wants to break the security of HMAC outputs the message $m=(N, R, c)$ and $\sigma=\operatorname{HMAC}_{k}(m)$. This leads to a contradiction under the security of HMAC.

2) If $\mathcal{A}$ impersonates $T_{c}$ 's owner: this yields that $\mathcal{A}$ sends a nonce $N$ to $T_{c}$. Then, $\mathcal{A}$ receives a ciphertext $c$, a random number $R$ and $\sigma=\operatorname{HMAC}_{k}(N, R, c)$ from tag $T_{c}$. Finally, $\mathcal{A}$ replies with a ciphertext $c^{\prime}$ and $\sigma^{\prime}=\operatorname{HMAC}_{k}\left(R, c^{\prime}\right)$.

An adversary $\mathcal{A}^{\prime}$ who wants to break the security of HMAC outputs the message $m^{\prime}=R \| c^{\prime}$ and $\sigma^{\prime}=\operatorname{HMAC}_{k}\left(m^{\prime}\right)$. This leads to a contradiction under the security of HMAC.

Theorem 4 (Exclusive ownership). The ownership transfer protocol in $R O$ $T I V$ provides exclusive ownership under the security of hash function $H$.

Proof (sketch). Assume there is an adversary $\mathcal{A}$ who breaks the exclusive ownership of ROTIV with a non negligible advantage. We construct an adversary $\mathcal{A}^{\prime}$ who breaks the one wayness of the hash function $H$ with a non negligible advantage.

Let $\mathcal{A}^{\prime}$ denotes an adversary against the one wayness of $H$. That is, given $h=H(t), \mathcal{A}^{\prime}$ outputs $t$.

To break the one wayness of $H, \mathcal{A}^{\prime}$ writes a valid state $s_{\left(T_{c}, j\right)}=\left(k_{\left(T_{c}, j\right)}, c_{\left(T_{c}, j\right)}\right)$ into the challenge tag $T_{c}$, such that $c_{\left(T_{c}, j\right)}$ is an encryption of $h^{x}=H(t)^{x}$. At the end of the challenge phase of the exclusive ownership experiment, $\mathcal{A}$ is required to transfer the ownership of tag $T_{c}$ to a challenge owner $O_{c}$.

If $\mathcal{A}$ 's advantage in the exclusive ownership experiment is non-negligible, then this means that $\mathcal{A}$ is able to supply $O_{c}$ with valid ownership references of tag $T_{c}: \operatorname{ref}_{T_{c}}^{O}=\left(t, h^{x}, k_{\text {old }}, k_{\text {new }}\right)$ with non negligible advantage. 
To break the one wayness of $H, \mathcal{A}^{\prime}$ outputs $t$ as provided by $\mathcal{A}$. This leads to a contradiction under the one wayness of $H$.

Theorem 5 (Issuer verification security). The issuer verification protocol in ROTIV is secure under the BCDH assumption.

Proof (sketch). Assume there is an adversary $\mathcal{A}$ who can break the security of the issuer verification protocol with a non-negligible advantage. We build an adversary $\mathcal{A}^{\prime}$ who breaks the $\mathrm{BCDH}$ assumption, that is, given $g_{1}, g_{1}^{x}, g_{1}^{y}, g_{1}^{z} \in$ $\mathbb{G}_{1}$ and $g_{2}, g_{2}^{x}, g_{2}^{y} \in \mathbb{G}_{2}$ for random $x, y, z \in \mathbb{F}_{q}$, the probability to compute $e\left(g_{1}, g_{2}\right)^{x y z}$ is negligible.

To break BCDH assumption, $\mathcal{A}^{\prime}$ simulates an issuer of ROTIV whose secret key is $s k=x$ and public key $p k=g_{2}^{x}$. $\mathcal{A}^{\prime}$ also simulates the output of the hash function $H$ during the issuer verification experiment. In the challenge phase, when $\mathcal{A}$ creates a tag $T_{c}$, he selects a random identifier $t_{c}$. Then, $\mathcal{A}$ queries $H$ with $t_{c}$. To compute $H\left(t_{c}\right), \mathcal{A}^{\prime}$ selects randomly $r_{c} \in \mathbb{F}_{q}$ and returns $h_{c}=$ $H\left(t_{c}\right)=g_{1}^{z r_{c}}$.

If $\mathcal{A}$ has a non-negligible advantage in breaking the issuer verification security, then this yields that $\mathcal{A}$ is able to output valid verification references for $T_{c}$, $\operatorname{ref}_{T_{c}}^{V}=\left(A_{c}, B_{c}, C_{c}\right)=\left(t_{c}, h_{c}^{x}, C_{c}\right)=\left(t_{c}, g_{1}^{x z r_{c}}, C_{c}\right)$.

Therefore, to break BCDH $\mathcal{A}^{\prime}$ outputs $e\left(g_{1}, g_{2}\right)^{x y z}=e\left(g_{1}^{x z r_{c}}, g_{2}^{y}\right)^{r_{c}^{-1}}$.

\section{Related Work}

Molnar et al. [14] address the problem of ownership transfer in RFID systems by using tag pseudonyms and relying on a trusted third party. Here, the TTP is the only entity than can identify tags. To transfer ownership of tag $T$, the current owner of $T O_{(T, k)}$, and the prospective owner of $T O_{(T, k+1)}$, contact the TTP, who then provides $O_{(T, k+1)}$ with $T$ 's identity. Once the ownership transfer of $T$ takes place, the TTP refuses identity requests from $T$ 's previous owner $O_{(T, k)}$. However, relying on a TTP is a drawback: in many scenarios, the availability of a trusted third party during tag ownership transfer is probably unrealistic.

Other solutions based on symmetric primitives have been proposed by Lim and Kwon [13], Fouladgar and Afifi [7], Song [18], and Kulseng et al. [1]]. These schemes however suffer as discussed in section 2.2 from three major drawbacks: 1.) tag identification and authentication is linear in the number of tags, 2.) desynchronization and 3.) no tag issuer verification.

Kapoor and Piramuthu [10] suggests a two party ownership transfer protocol based on keyed hash functions. In order to provide forward unlinkability, the new owner of tag $T, O_{(T, k+1)}$ does not have access to the key of the previous owner $O_{(T, k+1)}$. Also, to cope with desynchronization, $T$ 's owner does not update the shared key unless he receives an acknowledgment from $T$. However, as the scheme relies on symmetric primitives it still suffers from linear time authentication and lack of issuer verification.

Dimitrou [5] proposes a solution to ownership transfer that relies on symmetric cryptography while relaxing the privacy requirements for both backward 
and forward unlinkability. Unlike previous schemes on ownership transfer, this solution allows an owner of a tag to revert the tag to its original state. This is useful for after sales services where a retailer can recognize a sold tag $T$. Note that ROTIV offers the same feature: a tag $T$ 's unique identifier will allow any owner to verify whether he owned $T$ before or not.

\section{Conclusion}

In this paper, we presented ROTIV to address security and privacy issues related to RFID ownership transfer in supply chains. Moreover, ROTIV enables ownership transfer together with issuer verification. Such verification will prevent partners in a supply chain from injecting fake products. ROTIV's main idea is to store a signature of the issuer in tags that can be verified by every partner in the supply chain. Also, to allow for efficient ownership transfer, ROTIV comprises an efficient, constant time authentication protocol. To guarantee tag privacy, we use re-encryption and key update techniques. Despite the high security and privacy properties, ROTIV is lightweight and requires a tag to only evaluate a hash function.

\section{References}

[1] Ateniese, G., Camenisch, J., de Medeiros, B.: Untraceable rfid tags via insubvertible encryption. In: CCS 2005: Proceedings of the 12th ACM Conference on Computer and Communications Security, pp. 92-101. ACM, New York (2005) ISBN 1-59593-226-7

[2] Ateniese, G., Kirsch, J., Blanton, M.: Secret handshakes with dynamic and fuzzy matching. In: Proceedings of the Network and Distributed System Security Symposium, NDSS. The Internet Society (2007)

[3] Ballard, L., Green, M., de Medeiros, B., Monrose, F.: Correlation-resistant storage via keyword-searchable encryption. In: Cryptology ePrint Archive, Report 2005/417 (2005), http://eprint.iacr.org/

[4] Burmester, M., de Medeiros, B., Motta, R.: Robust, anonymous RFID authentication with constant key-lookup. In: Proceedings of the 2008 ACM Symposium on Information, Computer and Communications Security, ASIACCS 2008, pp. 283-291. ACM, New York (2008) ISBN 978-1-59593-979-1

[5] Dimitrou, T.: rfidDOT: RFID delegation and ownership transfer made simple. In: Proceedings of International Conference on Security and Privacy in Communication Networks, Istanbul, Turkey (2008) ISBN 978-1-60558-241-2

[6] Elkhiyaoui, K., Blass, E.-O., Molva, R.: ROTIV: RFID Ownership Transfer with Issuer Verification. In: Cryptology ePrint Archive, Report 2010/634 (2010), http://eprint.iacr.org/

[7] Fouladgar, S., Afifi, H.: An Efficient Delegation and Transfer of Ownership Protocol for RFID Tags. In: First International EURASIP Workshop on RFID Technology, Vienna, Austria (September 2007)

[8] Galbraith, S.D., Paterson, K.G., Smart, N.P.: Pairings for cryptographers. Discrete Appl. Math. 156, 3113-3121 (2008) ISSN 0166-218X 
[9] Juels, A., Weis, S.A.: Defining Strong Privacy for RFID. In: PerCom Workshops, White Plains, USA, pp. 342-347 (2007) ISBN 978-0-7695-2788-8

[10] Kapoor, G., Piramuthu, S.: Single RFID Tag Ownership Transfer Protocols. IEEE Transactions on Systems, Man, and Cybernetics 99, 1-10 (2011) ISSN 1094-6977

[11] Kulseng, L., Yu, Z., Wei, Y., Guan, Y.: Lightweight mutual authentication and ownership transfer for rfid systems. In: INFOCOM, pp. 251-255 (2010)

[12] Lee, Y.K., Batina, L., Singelée, D., Verbauwhede, I.: Low-Cost Untraceable Authentication Protocols for RFID. In: Wetzel, S., Nita-Rotaru, C., Stajano, F.:Proceedings of the 3rd ACM Conference on Wireless Network Security - WiSec 2010, Hoboken, New Jersey, USA, pp. 55-64. ACM, ACM Press (March 2010)

[13] Lim, C.H., Kwon, T.: Strong and Robust RFID Authentication Enabling Perfect Ownership Transfer. In: Ning, P., Qing, S., Li, N. (eds.) ICICS 2006. LNCS, vol. 4307, pp. 1-20. Springer, Heidelberg (2006)

[14] Molnar, D., Soppera, A., Wagner, D.: A Scalable, Delegatable Pseudonym Protocol Enabling Ownership Transfer of RFID Tags. In: Preneel, B., Tavares, S. (eds.) SAC 2005. LNCS, vol. 3897, pp. 276-290. Springer, Heidelberg (2006)

[15] Ohkubo, M., Suzuki, K., Kinoshita, S.: Cryptographic Approach to "PrivacyFriendly" Tags. In: RFID Privacy Workshop. MIT, Massachusetts (2003)

[16] Paise, R., Vaudenay, S.: Mutual authentication in RFID: security and privacy. In: Proceedings of the 2008 ACM Symposium on Information, Computer and Communications Security, ASIACCS 2008, pp. 292-299. ACM, New York (2008) ISBN 978-1-59593-979-1

[17] Saito, J., Imamoto, K., Sakurai, K.: Reassignment Scheme of an RFID Tag's Key for Owner Transfer. In: Enokido, T., Yan, L., Xiao, B., Kim, D.Y., Dai, Y.S., Yang, L.T. (eds.) EUC-WS 2005. LNCS, vol. 3823, pp. 1303-1312. Springer, Heidelberg (2005)

[18] Song, B.: RFID Tag Ownership Transfer. In: Workshop on RFID Security - RFIDSec 2008, Budapest, Hungary (July 2008)

[19] Vaudenay, S.: On Privacy Models for RFID. In: Kurosawa, K. (ed.) ASIACRYPT 2007. LNCS, vol. 4833, pp. 68-87. Springer, Heidelberg (2007) ISBN 3-540-768998, 978-3-540-76899-9 